Bull. Soc. math. France

130 (4), 2002, p. $573-585$

\title{
LES SHIFTS À POIDS DISSYMÉTRIQUES SONT HYPER-RÉFLEXIFS
}

\author{
PAR XAVIER DUSSAU
}

RÉSumÉ. - Nous prouvons l'hyper-réflexivité du shift bilatéral $S_{\omega}$ sur $\ell_{\omega}^{2}(\mathbb{Z})$, lorsque le poids vérifie $\omega(n)=1$ for $n \geq 0$ et $\lim _{n \rightarrow-\infty} \omega(n)=+\infty$.

ABSTRACT (Dissymmetric bilateral weighted shifts are hyper-reflexive)

We prove the hyper-reflexivity of the bilateral weighted shift $S_{\omega}$ on $\ell_{\omega}^{2}(\mathbb{Z})$, when the weight satisfies $\omega(n)=1$ for $n \geq 0$, and $\lim _{n \rightarrow-\infty} \omega(n)=+\infty$.

\section{Introduction}

On appellera poids toute application $\omega: \mathbb{Z} \rightarrow] 0,+\infty[$ vérifiant

$$
0<\inf _{n \in \mathbb{Z}} \frac{\omega(n+1)}{\omega(n)} \leq \sup _{n \in \mathbb{Z}} \frac{\omega(n+1)}{\omega(n)}<+\infty .
$$

Étant donné un poids $\omega$, on introduit l'espace

$$
\ell_{\omega}^{2}(\mathbb{Z}):=\left\{u=\left(u_{n}\right)_{n \in \mathbb{Z}} ;\|u\|_{\omega}=\left[\sum_{n \in \mathbb{Z}}\left|u_{n}\right|^{2} \omega(n)^{2}\right]^{1 / 2}<\infty\right\} .
$$

Muni du produit scalaire $[u, v]=\sum_{n \in \mathbb{Z}} u_{n} \cdot \overline{v_{n}} \omega(n)^{2}, \ell_{\omega}^{2}(\mathbb{Z})$ est un espace de Hilbert.

Texte reçu le 20 juillet 2001, accepté le 14 février 2002

Xavier Dussau, Laboratoire de Mathématiques Pures, Université Bordeaux I, 351, cours de la Libération, 33405 Talence Cedex (France) • E-mail : dussau@math.u-bordeaux.fr Classification mathématique par sujets (2000). — 47B37.

Mots clefs. - Shift à poids, reflexivité. 
Considérons dans $\ell_{\omega}^{2}(\mathbb{Z})$ le shift

$$
S_{\omega}:\left(u_{n}\right)_{n \in \mathbb{Z}} \longmapsto\left(u_{n-1}\right)_{n \in \mathbb{Z}} .
$$

Cet opérateur est clairement borné et inversible, et pour tout $n \in \mathbb{Z}$,

$$
\left\|S_{\omega}^{n}\right\|=\sup _{p \in \mathbb{Z}} \frac{\omega(n+p)}{\omega(p)} .
$$

Un poids $\omega$ est dit dissymétrique si

$$
\omega(n)=1 \text { pour } n \geq 0 \text { et } \lim _{n \rightarrow-\infty} \omega(n)=+\infty .
$$

Si $H$ est un espace de Hilbert séparable, on peut identifier $\mathcal{B}(H)$ au dual de l'espace $\mathcal{C}^{1}(H)$ des opérateurs à trace, la dualité étant obtenue par la formule $\langle N, T\rangle=\operatorname{Tr}(T N)$ pour $N \in \mathcal{C}^{1}(H), T \in \mathcal{B}(H)$. Ceci permet de considérer la topologie $w^{*}$ sur $\mathcal{B}(H)$. L'algèbre $w^{*}$-fermée engendrée par un opérateur $V$ sera notée $\mathcal{A}_{V}$.

Un opérateur $V \in \mathcal{B}(H)$ est dit réflexif si tout opérateur qui laisse invariant les sous-espaces fermés de $H$ stables par $V$ appartient à $\mathcal{A}_{V}$.

On note $\{V\}^{\prime}$ le commutant de $V$.

Un sous-espace fermé $F$ de $H$ est hyper-invariant pour $V$ si $R(F) \subset F$ pour tout $R \in\{V\}^{\prime}$.

On dit que $V$ est hyper-réflexif si tout opérateur qui laisse stable les sousespaces hyper-invariants de $V$, commute avec $V$.

Nous montrons dans cet article le résultat suivant.

THÓRÈme 1. - Si $\omega$ est un poids dissymétrique, le shift $S_{\omega}$ sur $\ell_{\omega}^{2}(\mathbb{Z})$ est hyper-réflexif.

D'autres résultats d'hyper-réflexivité pour les shifts bilatéraux ont été obtenus récemment. L'auteur de cet article a prouvé dans [3], l'hyper-réflexivité du shift lorsque le poids vérifie la condition :

$$
1 \leq \omega(n) \leq C(1+|n|)^{\alpha} \quad \text { pour } \quad n \in \mathbb{Z}
$$

le réel $\alpha$ étant une constante strictement positive. Ce cas m'avait été suggéré par J. Esterle, qui précédemment (voir [4]), avait montré l'hyper-réflexivité du shift sur certains poids très irréguliers (poids d'Apostol). Notons que l'hyperréflexivité de $S_{\omega}$ entraîne la réflexivité de $S_{\omega}$ et $S_{\omega}^{-1}$ (voir la remarque 2 de [4]).

Un sous-espace fermé $M$ de $\ell_{\omega}^{2}(\mathbb{Z})$ sera dit invariant par translation si $S_{\omega}(M) \cup S_{\omega}^{-1}(M) \subset M$. Comme le commutant de $S_{\omega}$ est l'algèbre $w^{*}$-fermée engendrée par $S_{\omega}$ et $S_{\omega}^{-1}(\operatorname{voir}[9])$, un sous-espace fermé $F$ de $\ell_{\omega}^{2}(\mathbb{Z})$ est hyper-invariant pour $S_{\omega}$ seulement si il est invariant par translation.

Un sous-espace invariant par translation $F$ qui vérifie $F \neq\{0\}$ et $F \neq \ell_{\omega}^{2}(\mathbb{Z})$ est dit non trivial (noté SEINT).

TOME $130-2002-\mathrm{N}^{\mathrm{O}} 4$ 
Soit $\omega$ un poids dissymétrique. Considérons

$$
L_{\omega}^{2}(\mathbb{T}):=\left\{f \in L^{2}(\mathbb{T}) ;\|f\|_{\omega}=\left[\sum_{n \in \mathbb{Z}}|\widehat{f}(n)|^{2} \cdot \omega(n)^{2}\right]^{1 / 2}<\infty\right\} .
$$

Alors l'application $\mathcal{F}$ de $L^{2}(\mathbb{T})$ dans $\ell^{2}(\mathbb{Z})$ qui à $f$ associe $(\widehat{f}(n))_{n}$ induit un isomorphisme isométrique entre $L_{\omega}^{2}(\mathbb{T})$ et $\ell_{\omega}^{2}(\mathbb{Z})$. De plus l'opérateur $S_{\omega}^{\prime}$ : $f\left(\mathrm{e}^{i \theta}\right) \mapsto \mathrm{e}^{i \theta} f\left(\mathrm{e}^{i \theta}\right)$ sur $L_{\omega}^{2}(\mathbb{T})$ est unitairement équivalent au shift $S_{\omega} \operatorname{sur} \ell_{\omega}^{2}(\mathbb{Z})$. Soit $f \in L_{\omega}^{2}(\mathbb{T}), f \neq 0 ;$ alors $\left.E(f):=\left(\operatorname{vect}_{n \in \mathbb{Z}} T_{\omega}^{n} f\right)^{-}\right)$est un sous-espace hyper-invariant pour l'opérateur $S_{\omega}^{\prime}$. Ainsi $\widehat{E(f)}:=\mathcal{F}(E(f))$ est un SEINT si $E(f) \neq L_{\omega}^{2}(\mathbb{T})$.

J. Esterle a prouvé l'existence de SEINT dans le cas dissymétrique (voir [6]), en exhibant une fonction intérieure $U$ telle que $E(U) \neq L_{\omega}^{2}(\mathbb{T})$.

La proposition suivante, qui sera démontrée au paragraphe 3, est le point de départ de notre article.

Proposition 1. - Soit $M$ un SEINT pour le shift $S_{\omega}$ sur $\ell_{\omega}^{2}(\mathbb{Z})$. Si l'opérateur $\widetilde{S}_{\omega}$ sur $\ell_{\omega}^{2}(\mathbb{Z}) / M$ défini par $\widetilde{S}_{\omega}(f+M)=S_{\omega}(f)+M$ est hyper-réflexif, alors $S_{\omega}$ est hyper-réflexif.

Ainsi, il suffit de trouver un SEINT $M$ pour lequel $\widetilde{S}_{\omega}$ est hyper-réflexif, pour obtenir l'hyper-réflexivité de $S_{\omega}$.

Si $E(U) \neq L_{\omega}^{2}(\mathbb{T})$, il découle de la proposition 3.8 de [6] que l'opérateur

$$
\Phi: f+U H^{2}(\mathbb{D}) \longrightarrow f+E(U)
$$

est une injection de $H^{2}(\mathbb{D}) / U H^{2}(\mathbb{D})$ dans $L_{\omega}^{2}(\mathbb{T}) / E(U)$. Si

$$
\lim _{n \rightarrow+\infty} \frac{\log \omega(-n)}{\sqrt{n}}=+\infty
$$

$\phi$ est en plus inversible (voir prop. 3.8) et les opérateurs

$$
\widetilde{S}_{\omega}: f+\widehat{E(U)} \rightarrow S_{\omega} f+\widehat{E(U)} \quad \text { et } \quad M_{U}: f+U H^{2}(\mathbb{D}) \rightarrow S f+U H^{2}(\mathbb{D})
$$

sont semblables. Grâce à l'article de V.V. Kapustin [7], nous connaissons un critère pour l'hyper-réflexivité de $M_{U}$; ceci permet d'obtenir facilement l'hyperréflexivité de $S_{\omega}$ lorsque $\lim _{n \rightarrow+\infty} \log \omega(-n) / \sqrt{n}=+\infty$.

Le point délicat est donc d'obtenir l'inversibilité de $\Phi$ sans l'hypothèse supplémentaire ci-dessus. On va montrer que si $\omega$ est dissymétrique, il existe toujours une mesure positive singulière $\nu$ sur le cercle unité qui s'annule sur les ensembles de Carleson, telle que $\Phi: H^{2}(\mathbb{D}) / U H^{2}(\mathbb{D}) \rightarrow L_{\omega}^{2}(\mathbb{T}) / E(U)$ soit bijective ( $U$ désignant la fonction intérieure associée à $\nu$ ). Il faut pour cela étudier en détail $U H^{2}(\mathbb{D})^{\perp}$ car la méthode de [6], prop. 3.8, utilisée pour montrer la surjectivité de $\Phi$ quand $\lim _{n \rightarrow+\infty} \log \omega(-n) / \sqrt{n}=+\infty$ fait appel aux inégalités de Cauchy et ne permet pas de conclure lorsque $\sum_{n=1}^{\infty} 1 / \omega^{2}(-n)=+\infty$.

BULletin DE LA SOCIÉtÉ MATHÉMATIQUE DE FRANCE 


\section{Préliminaires}

Dans cet article, $\mathbb{D}$ et $\mathbb{T}$ désigneront respectivement le disque unité ouvert et le cercle unité de $\mathbb{C}$. L'espace $\left\{f \in L_{\omega}^{2}(\mathbb{T}) ; \widehat{f}(n)=0\right.$ pour $\left.n<0\right\}$ s'identifie à l'espace de Hardy $H^{2}(\mathbb{D})$, qui est muni de la norme

$$
\|f\|_{2}=\left[\sup _{0<r<1} \frac{1}{2 \pi} \int_{-\pi}^{\pi}\left|f\left(r \mathrm{e}^{i t}\right)\right|^{2} \mathrm{~d} t\right]^{1 / 2} .
$$

Si $f \in H^{2}(\mathbb{D})$, on note $f_{*} \in L^{2}(\mathbb{T})$ la fonction déterminée par les limites radiales de $f$. L'espace des fonctions holomorphes et bornées sur $\mathbb{D}$ est noté $H^{\infty}(\mathbb{D})$. Soit $U$ une fonction intérieure singulière, c'est à dire une fonction intérieure qui ne s'annule pas sur $\mathbb{D}$. Alors il existe une mesure $\mu$ positive singulière sur $\mathbb{T}$ telle que

$$
U(z)=\exp \left[-\frac{1}{2 \pi} \int_{\mathbb{T}} \frac{\xi+z}{\xi-z} \mathrm{~d} \mu(\xi)\right] \quad \text { pour } \quad z \in \mathbb{D} .
$$

Pour $0 \leq r<1$, on pose

$$
K_{U}(r)=\sup _{\theta \in[-\pi, \pi]} \frac{1}{\left|U\left(r \mathrm{e}^{i \theta}\right)\right|} .
$$

Comme dans [6], nous allons devoir utiliser les hyperdistributions que nous redéfinissons ci-dessous.

On appelle hyperdistribution, toute fonction analytique $\phi$ sur $\mathbb{C} \backslash \mathbb{T}$ telle que $\lim _{|z| \rightarrow+\infty} \phi(z)=0$. On désigne par $\operatorname{HD}(\mathbb{T})$, l'ensemble de toutes les hyperdistributions. Soit $\phi \in \operatorname{HD}(\mathbb{T})$; alors on pose $\phi^{+}=\phi_{\mid \mathbb{D}}, \phi^{-}=\phi_{|\mathbb{C}| \overline{\mathbb{D}}}$. Les coefficients de Fourier de $\phi$ sont définis de la façon suivante.

$$
\phi(z)= \begin{cases}+\sum_{n=1}^{\infty} \widehat{\phi}(n) z^{n-1} & \text { si }|z|<1, \\ -\sum_{n \leq 0} \widehat{\phi}(n) z^{n-1} & \text { si }|z|>1\end{cases}
$$

On pose

$$
\operatorname{HD}^{(2)}(\mathbb{T}):=\left\{\phi \in \operatorname{HD}(\mathbb{T}) ; \sum_{n<0}|\widehat{\phi}(n)|^{2}<+\infty\right\} .
$$

Soit $U$ une fonction intérieure singulière et soit $\mu$ la mesure associée à $U$. On pose $U(\infty)=\lim _{|z| \rightarrow+\infty} U(z)$. On définit $U^{*} \in \operatorname{HD}(\mathbb{T})$ par la formule

$$
U^{*}(z)=\frac{1}{U(z)}-\frac{1}{U(\infty)} \quad \text { pour } \quad z \in \mathbb{C} \backslash \mathbb{T} .
$$

On remarque que $U^{*} \in \mathrm{HD}^{(2)}(\mathbb{T})$ car $1 / U$ est bornée sur $\mathbb{C} \backslash \overline{\mathbb{D}}$.

Soit $\omega$ un poids dissymétrique. On pose

$$
\operatorname{HD}_{\omega}(\mathbb{T}):=\left\{\phi \in \operatorname{HD}(\mathbb{T}) ;\|\phi\|_{\omega}^{*}=\left[\sum_{n \in \mathbb{Z}} \frac{|\widehat{\phi}(n)|^{2}}{\omega^{2}(-n)}\right]^{1 / 2}<+\infty\right\} .
$$

TOME $130-2002-\mathrm{N}^{\mathrm{O}} 4$ 
On peut identifier $\operatorname{HD}_{\omega}(\mathbb{T})$ au dual de $L_{\omega}^{2}(\mathbb{T})$ en posant pour $f \in L_{\omega}^{2}(\mathbb{T}), \phi \in$ $\mathrm{HD}_{\omega}(\mathbb{T})$

$$
\langle f, \phi\rangle=\sum_{n \in \mathbb{Z}} \widehat{f}(n) \cdot \widehat{\phi}(-n)
$$

D'après [6], p. 80, nous avons

$$
\langle f, \phi\rangle=\lim _{r \rightarrow 1^{-}} \frac{1}{2 i \pi} \int_{\mathbb{T}} f(\xi)\left[\phi^{+}(r \xi)-\phi^{-}\left(\frac{\xi}{r}\right)\right] \mathrm{d} \xi .
$$

On pose

$$
E(U)^{\perp}=\left\{\phi \in \mathrm{HD}_{\omega}(\mathbb{T}) ;\langle f, \phi\rangle=0 \text { pour } f \in E(U)\right\} .
$$

Si $U^{*} \in \operatorname{HD}_{\omega}(\mathbb{T})$, nous avons en plus $U^{*} \in E(U)^{\perp}$ (voir [6], lemme 3.7). Ceci implique que $E(U) \neq L_{\omega}^{2}(\mathbb{T})$ et donc $\widehat{E(U)}$ est un SEINT de $\ell_{\omega}^{2}(\mathbb{Z})$. Posons

$$
\begin{aligned}
H_{0}^{2}(\mathbb{C} \backslash \overline{\mathbb{D}}):=\{u \in H(\mathbb{C} \backslash \overline{\mathbb{D}}) ; u(z) & =\sum_{n \leq 0} \widehat{u}(n) z^{n-1} \\
& \text { et } \left.\quad\|u\|_{2}=\left[\sum_{n \leq 0}|\widehat{u}(n)|^{2}\right]^{1 / 2}<+\infty\right\} .
\end{aligned}
$$

Soit $u \in H_{0}^{2}(\mathbb{C} \backslash \overline{\mathbb{D}})$; alors $\lim _{r \rightarrow 1^{-}} u(\xi / r)$ existe pour presque tout $\xi \in \mathbb{T}$. La fonction limite qui appartient à $L^{2}(\mathbb{T})$, est notée $u_{*}$. Le dual de $H^{2}(\mathbb{D})$ s'identifie à $H_{0}^{2}(\mathbb{C} \backslash \overline{\mathbb{D}})$ par la dualité

$$
\langle f, u\rangle=\sum_{n \geq 0} \widehat{f}(n) \cdot \widehat{u}(-n) .
$$

On remarque que

$$
\langle f, u\rangle=\frac{1}{2 \pi} \int_{-\pi}^{\pi} f_{*}\left(\mathrm{e}^{i t}\right) u_{*}\left(\mathrm{e}^{i t}\right) \mathrm{d} t
$$

et que cette dualité est la restriction de celle existant entre $L_{\omega}^{2}(\mathbb{T})$ et $\mathrm{HD}_{\omega}(\mathbb{T})$. On définit

$$
U H^{2}(\mathbb{D})^{\perp}=\left\{\phi \in H_{0}^{2}(\mathbb{C} \backslash \overline{\mathbb{D}}) ;\langle f, \phi\rangle=0 \text { pour } f \in U H^{2}(\mathbb{D})\right\} .
$$

Le résultat suivant est classique [2], mais nous en donnons une preuve pour la commodité du lecteur.

Proposition. - Soit $u \in H_{0}^{2}(\mathbb{C} \backslash \overline{\mathbb{D}})$; alors $u \in U H^{2}(\mathbb{D})^{\perp}$ si et seulement si il existe $g \in H^{2}(\mathbb{D})$ tel que

$$
\mathrm{e}^{i t} g_{*}\left(\mathrm{e}^{i t}\right)=U_{*}\left(\mathrm{e}^{i t}\right) u_{*}\left(\mathrm{e}^{i t}\right) \quad p \cdot p .
$$

BULletin DE LA SOCIÉtÉ MATHÉMATIQUE DE FRANCE 
Démonstration. - Étant donné $u \in H_{0}^{2}(\mathbb{C} \backslash \overline{\mathbb{D}})$,

$$
\begin{aligned}
u \in U H^{2}(\mathbb{D})^{\perp} & \Longleftrightarrow\left\langle U z^{n}, u\right\rangle=0, \quad \forall n \geq 0 \\
& \Longleftrightarrow \frac{1}{2 \pi} \int_{-\pi}^{\pi} \mathrm{e}^{-i t} U_{*}\left(\mathrm{e}^{i t}\right) u_{*}\left(\mathrm{e}^{i t}\right) \mathrm{e}^{-i n t} \mathrm{~d} t=0, \quad \forall n<0 \\
& \Longleftrightarrow \exists g \in H^{2}(\mathbb{D}) \text { tel que } \mathrm{e}^{i t} g_{*}\left(\mathrm{e}^{i t}\right)=U_{*}\left(\mathrm{e}^{i t}\right) u_{*}\left(\mathrm{e}^{i t}\right) \quad p . p .
\end{aligned}
$$

Soit $\mathcal{F}$ l'ensemble des fonctions $\sigma:[0,1[\rightarrow] 0,+\infty[$, telles que

$$
\frac{1}{\sigma} \in L^{2}([0,1]) \quad \text { et } \quad \int_{0}^{1} \frac{\mathrm{d} r}{\sigma^{2}(r)}=\frac{1}{2} .
$$

Étant donné $\sigma \in \mathcal{F}$, on pose

$$
\omega_{\sigma}(n)=1 \text { pour } n \geq 0 \quad \text { et } \quad \omega_{\sigma}(n)=\left[2 \int_{0}^{1} \frac{r^{-2 n}}{\sigma^{2}(r)} \mathrm{d} r\right]^{-1 / 2} \text { pour } n \leq 0 .
$$

Alors $\omega_{\sigma}$ est un poids dissymétrique, log-concave (ce qui signifie que la suite $\left(\omega_{\sigma}(-n-1) / \omega_{\sigma}(-n)\right)_{n \geq 0}$ est décroissante). On a, pour $\phi \in \mathrm{HD}_{\omega_{\sigma}}(\mathbb{T})$

$$
\|\phi\|_{\omega_{\sigma}}^{*}=\frac{1}{\pi} \iint_{\mathbb{D}} \frac{\left|\phi^{+}(\xi)\right|^{2} \cdot|\xi|}{\sigma^{2}(|\xi|)} \mathrm{d} s(\xi)+\sum_{n \leq 0}|\widehat{\phi}(n)|^{2},
$$

$\mathrm{d} s$ désignant la mesure planaire sur $\mathbb{C}$. Étant donné un poids dissymétrique $\omega$, on pose :

$$
\mathcal{G}(\omega)=\left\{\sigma \in \mathcal{F} ; \sup _{n<0} \frac{\omega_{\sigma}(n)}{\omega(n)}<+\infty\right\} .
$$

Dans le lemme 5.1 de [6], il est prouvé que pour tout poids dissymétrique décroissant $\omega$, il existe un poids dissymétrique log-concave $\bar{\omega}$ tel que $\sup _{n<0} \bar{\omega}(n) / \omega(n)<+\infty$. La décroissance de $\omega$ n'étant pas utilisée dans la preuve du lemme 5.1, le résultat est vrai pour tout poids dissymétrique.

De plus, si $\bar{\omega}$ est un poids dissymétrique log-concave, il existe $\sigma \in \mathcal{F}$ tel que

$$
0<\inf _{n<0} \frac{\omega_{\sigma}(n)}{\bar{\omega}(n)} \leq \sup _{n<0} \frac{\omega_{\sigma}(n)}{\bar{\omega}(n)}<+\infty
$$

(voir le lemme 5.2 de [6]). Ainsi, pour tout poids $\omega$ dissymétrique, l'ensemble $\mathcal{G}(\omega)$ est non vide.

\section{Réduction du problème}

Proposition 1. - Soit $M$ un SEINT pour le shift $S_{\omega}$ sur $\ell_{\omega}^{2}(\mathbb{Z})$. Si l'opérateur $\widetilde{S}_{\omega}$ sur $\ell_{\omega}^{2}(\mathbb{Z}) / M$ défini par

$$
\widetilde{S}_{\omega}(f+M)=S_{\omega}(f)+M
$$

est hyper-réflexif, alors $S_{\omega}$ est hyper-réflexif.

TOME $130-2002-\mathrm{N}^{\mathrm{O}} 4$ 
Démonstration. - Soit $T$ un opérateur sur $\ell_{\omega}^{2}(\mathbb{Z})$ qui laisse stable les SEINT. Nous devons montrer que $S_{\omega} T=T S_{\omega}$. Introduisons l'opérateur

$$
\begin{aligned}
\widetilde{T}: \ell_{\omega}^{2}(\mathbb{Z}) / M & \longrightarrow \ell_{\omega}^{2}(\mathbb{Z}) / M, \\
f+M & \longmapsto T f+M .
\end{aligned}
$$

Soit $\pi: \ell_{\omega}^{2}(\mathbb{Z}) \rightarrow \ell_{\omega}^{2}(\mathbb{Z}) / M$ la surjection canonique.

Considérons un sous-espace fermé $G$ de $\ell_{\omega}^{2}(\mathbb{Z}) / M$ hyper-invariant pour $\widetilde{S}_{\omega}$. Soient $f \in \pi^{-1}(G)$ et $n \in \mathbb{Z}$; alors

$$
\pi\left(S_{\omega}^{n} f\right)=\widetilde{S}_{\omega}^{n}(\pi(f)) \in G .
$$

Ainsi $\pi^{-1}(G)$ est hyperinvariant pour $S_{\omega}$. On en déduit que $T\left(\pi^{-1}(G)\right) \subset$ $\pi^{-1}(G)$ et donc $\widetilde{T}(G) \subset G$. Par conséquent $\widetilde{T}$ laisse stable tous les sous-espaces hyper-invariants de $\widetilde{S}_{\omega}$.

Puisque $\widetilde{S}_{\omega}$ est hyper-réflexif, $\widetilde{S}_{\omega}$ et $\widetilde{T}$ commutent et donc $S_{\omega} T f-T S_{\omega} f \in M$ pour tout $f \in \ell_{\omega}^{2}(\mathbb{Z})$. Pour $\theta \in \mathbb{R}$, on pose :

$$
M_{\theta}:=\left\{u \in \ell_{\omega}^{2}(\mathbb{Z}) ;\left(u_{n} \mathrm{e}^{i n \theta}\right)_{n} \in M\right\} .
$$

Les $M_{\theta}$ sont des SEINT de $S_{\omega}$ et d'après le raisonnement ci-dessus

$$
S_{\omega} T f-T S_{\omega} f \in \bigcap_{\theta \in \mathbb{R}} M_{\theta} .
$$

$M$ est un SEINT, donc il existe $v \in \ell_{\omega}^{2}(\mathbb{Z})$, non nul, orthogonal à $M$. On choisit $n_{0} \in \mathbb{Z}$ tel que $v_{n_{0}} \neq 0$. Supposons qu'il existe $u \in \bigcap_{\theta \in \mathbb{R}} M_{\theta}$, non nul; comme $\bigcap_{\theta \in \mathbb{R}} M_{\theta}$ est invariant par translation, on peut supposer que $u_{n_{0}} \neq 0$. Pour $\theta \in \mathbb{R},\left(u_{n} \mathrm{e}^{i n \theta}\right)_{n} \in M$, donc $\sum_{n \in \mathbb{Z}} u_{n} \mathrm{e}^{i n \theta} \cdot \overline{v_{n}} \omega(n)^{2}=0$. On en conclut que $u_{n} \cdot \overline{v_{n}}=0$ pour $n \in \mathbb{Z}$. Or ceci est absurde car $u_{n_{0}}$ et $v_{n_{0}}$ sont non nuls. L'intersection des $M_{\theta}$ est donc réduite à $\{0\}$ et finalement $S_{\omega} T=T S_{\omega}$.

Grâce à la proposition précédente, il nous suffit de trouver un SEINT $M$ tel que $\widetilde{S}_{\omega}: f+M \rightarrow S_{\omega} f+M$ soit hyper-réflexif.

Soit $S$ le shift unilatéral sur $H^{2}(\mathbb{D})$. On définit l'opérateur $M_{U}$ sur $H^{2}(\mathbb{D}) / U H^{2}(\mathbb{D})$ par la formule

$$
M_{U}\left(f+U H^{2}(\mathbb{D})\right)=S f+U H^{2}(\mathbb{D}) .
$$

$M_{U}$ est une contraction (on a $\left\|M_{U}\right\| \leq 1$ ), complètement non unitaire (il n'existe pas de sous-espace fermé $F$ tel que $T_{\mid F}$ est unitaire) et $\sigma\left(M_{U}\right)$, le spectre de $M_{U}$, est inclus dans le support, au sens des distributions, de $\mu$ (voir [8]).

Lemme 1. - Soient $\omega$ un poids dissymétrique et $\sigma \in \mathcal{G}(\omega)$. Considérons une fonction intérieure singulière $U$ telle que $\int_{0}^{1} K_{U}(r)^{2} / \sigma^{2}(r) \mathrm{d} r<+\infty$. Soit $u$ dans $U H^{2}(\mathbb{D})^{\perp}$; alors il existe $v \in \mathrm{HD}_{\omega}(\mathbb{T})$ et $C>0$ tels que

$$
v^{-}=u, \quad\|v\|_{\omega}^{*} \leq C\|u\|_{2} \quad \text { et } \quad v \in E(U)^{\perp} .
$$

BULletin DE LA SOCiÉtÉ MATHÉmATiQUe DE FRANCE 
Démonstration. - En utilisant le résultat rappelé dans les préliminaires, on voit qu'il existe $g \in H^{2}(\mathbb{D})$ tel que $\mathrm{e}^{i t} g_{*}\left(\mathrm{e}^{i t}\right)=U_{*}\left(\mathrm{e}^{i t}\right) u_{*}\left(\mathrm{e}^{i t}\right)$ p.p. Soit $v$ l'hyperdistribution définie par

$$
v^{+}(z)=\frac{z g(z)}{U(z)} \quad \text { et } \quad v^{-}(z)=u(z)
$$

On a

$$
\left(\|v\|_{\omega}^{*}\right)^{2} \leq M\left(\|v\|_{\omega_{\sigma}}^{*}\right)^{2}=\frac{M}{\pi} \iint_{\mathbb{D}} \frac{|\xi g(\xi) / U(\xi)|^{2}|\cdot \xi|}{\sigma^{2}(|\xi|)} \mathrm{d} s(\xi)+M \sum_{n \leq 0}|\widehat{u}(n)|^{2} .
$$

De plus,

$$
\begin{aligned}
\frac{1}{\pi} \iint_{\mathbb{D}} \frac{|\xi g(\xi) / U(\xi)|^{2} \cdot|\xi|}{\sigma^{2}(|\xi|)} \mathrm{d} s(\xi) & \leq \frac{1}{\pi} \int_{0}^{1} \int_{-\pi}^{\pi} \frac{\left|g\left(r \mathrm{e}^{i \theta}\right)\right|^{2}}{\left|U\left(r \mathrm{e}^{i \theta}\right)\right|^{2} \cdot \sigma^{2}(r)} \mathrm{d} r \mathrm{~d} \theta \\
& \leq 2\left(\|g\|_{2}\right)^{2} \int_{0}^{1} \frac{K_{U}(r)^{2}}{\sigma^{2}(r)} \mathrm{d} r .
\end{aligned}
$$

Puisque $\|g\|_{2}=\|u\|_{2}=\left(\sum_{n \leq 0}|\widehat{u}(n)|^{2}\right)^{1 / 2}$, nous obtenons $\|v\|_{\omega}^{*} \leq C\|u\|_{2}$, et a fortiori $v \in \mathrm{HD}_{\omega}(\mathbb{T})$.

Il nous reste à montrer que $v \in E(U)^{\perp}$. Pour $n \in \mathbb{Z}$, nous avons

$$
\begin{aligned}
\left\langle z^{n} U, v\right\rangle & =\lim _{r \rightarrow 1^{-}} \frac{1}{2 i \pi} \int_{\mathbb{T}} \xi^{n} U_{*}(\xi)\left[\frac{g(r \xi)}{U(r \xi)}-\frac{\xi}{r} u\left(\frac{\xi}{r}\right)\right] \mathrm{d} \xi \\
& =\frac{1}{2 i \pi} \int_{\mathbb{T}} \xi^{n} g_{*}(\xi) \mathrm{d} \xi-\frac{1}{2 i \pi} \int_{\mathbb{T}} \xi^{n+1} U_{*}(\xi) u_{*}(\xi) d \xi
\end{aligned}
$$

grâce au théorème de convergence dominée.

Comme $\mathrm{e}^{i t} g_{*}\left(\mathrm{e}^{i t}\right)=U_{*}\left(\mathrm{e}^{i t}\right) u_{*}\left(\mathrm{e}^{i t}\right)$ p.p., on a $\left\langle z^{n} U, v\right\rangle=0$ pour $n \in \mathbb{Z}$, et donc $v \in E(U)^{\perp}$.

Proposition 2. - Soient $\omega$ un poids dissymétrique, $\sigma \in \mathcal{G}(\omega)$, et soit $U$ une fonction intérieure singulière telle que $\int_{0}^{1} K_{U}(r)^{2} / \sigma^{2}(r) \mathrm{d} r<+\infty$. Alors $\widehat{E(U)}$ est un SEINT, et les opérateurs $\widetilde{S}_{\omega}$ et $M_{U}$ définis respectivement sur $\ell_{\omega}^{2}(\mathbb{Z}) \widehat{E(U)}$ et $H^{2}(\mathbb{D}) / U H^{2}(\mathbb{D})$ sont semblables.

Démonstration. - Comme $\int_{0}^{1} K_{U}(r)^{2} / \sigma^{2}(r) \mathrm{d} r<+\infty, U^{*} \in \operatorname{HD}_{\omega_{\sigma}}(\mathbb{T}) \subset$ $\mathrm{HD}_{\omega}(\mathbb{T})$. Ainsi, $E(U) \neq L_{\omega}^{2}(\mathbb{T})$ et par conséquent $\widehat{E(U)}$ est un SEINT.

Soit $\Phi$ l'opérateur de $H^{2}(\mathbb{D}) / U H^{2}(\mathbb{D})$ dans $L_{\omega}^{2}(\mathbb{T}) / E(U)$ tel que

$$
\Phi\left(f+U H^{2}(\mathbb{D})\right)=f+E(U) .
$$

Montrons que l'image de $\Phi$ est dense dans $L_{\omega}^{2}(\mathbb{T}) / E(U)$. Il nous suffit de montrer que pour tout $n \in \mathbb{Z}, z^{n}+E(U)$ appartient à l'image de $\Phi$.

Si $n \geq 0, \Phi\left(z^{n}+U H^{2}\right)=z^{n}+E(U)$.

TOME $130-2002-\mathrm{N}^{\mathrm{O}} 4$ 
Si $n<0$, on pose

$$
V_{n}(z)=\left(\frac{1-U(0)^{-1} U(z)}{z}\right)^{-n}
$$

Alors $V_{n} \in H^{2}$ et $V_{n}(z)=z^{n}+z^{n} U(0)^{-1} U(z) g_{n}(z)$ où $g_{n} \in H^{2}(\mathbb{D})$. Ainsi $V_{n}(z)-z^{n} \in E(U)$ et donc $\Phi\left(V_{n}+U H^{2}(\mathbb{D})\right)=V_{n}+E(U)=z^{n}+E(U)$. L'image de $\Phi$ est donc dense.

Nous allons maintenant montrer que $\Phi$ est injectif et d'image fermée. Pour obtenir cela, il suffit de vérifier qu'il existe $C>0$ tel que pour tout $f \in H^{2}(\mathbb{D})$, $\left\|\pi_{\omega}(f)\right\| \geq C\left\|\pi_{U}(f)\right\|$. Nous avons

$$
\left\|\pi_{U}(f)\right\|=\sup _{\substack{\|u\| \leq 1 \\ u \in\left(U H^{2}(\mathbb{D})\right)^{\perp}}}|\langle f, u\rangle| .
$$

Soit $\epsilon>0$. Il existe $u \in\left(U H^{2}(\mathbb{D})\right)^{\perp},\|u\|_{2} \leq 1$ tel que

$$
|\langle f, u\rangle|>\left\|\pi_{U}(f)\right\|-\epsilon .
$$

D'après le lemme précédent, il existe $v \in E(U)^{\perp}$ tel que $v^{-}=u$ et $\|v\|_{\omega}^{*} \leq$ $K\|u\|_{2}$, où $K$ est une constante positive indépendante de $U$. Ainsi,

$$
\begin{aligned}
\left\|\pi_{U}(f)\right\|-\epsilon<|\langle f, u\rangle|=|\langle f, v\rangle| & \leq\left\|\pi_{\omega}(f)\right\| \cdot\|v\|_{\omega}^{*} \\
& \leq K\left\|\pi_{\omega}(f)\right\| \cdot\|u\|_{2} \\
& \leq K\left\|\pi_{\omega}(f)\right\| .
\end{aligned}
$$

Par conséquent, $\left\|\pi_{\omega}(f)\right\| \geq K^{-1}\left\|\pi_{U}(f)\right\|$. L'opérateur $\Phi$ est donc inversible. On en déduit alors facilement que les opérateurs $\widetilde{S}_{\omega}$ et $M_{U}$ sont semblables.

L'existence d'une fonction intérieure singulière $U$ vérifiant les hypothèses de la proposition précédente sera démontrée dans le prochain paragraphe.

Soient $T_{1}$ et $T_{2}$ deux opérateurs semblables sur un espace de Banach $X$; alors $T_{1}$ est hyper-réflexif si et seulement si $T_{2}$ est hyper-réflexif. L'hyper-réflexivité de $M_{U}$ entraînera donc l'hyper-réflexivité de $\tilde{T}_{\omega}$.

Soit $m$ la mesure de Lebesgue sur $\mathbb{T}$. Un sous-ensemble fermé $E$ inclus dans $\mathbb{T}$ est dit de Carleson lorsque

$$
m(E)=0 \quad \text { et } \quad \sum_{k} \ell_{k} \log \left(\frac{2 \pi}{\ell_{k}}\right)<+\infty
$$

où les $\ell_{k}$ sont les longueurs des arcs du complémentaire de $E$.

Rappelons qu'un élément $x$ d'une algèbre est dit idempotent si $x^{2}=x$.

V.V. Kapustin a montré dans [7] que les trois assertions suivantes sont équivalentes :

- $M_{U}$ est réflexif;

- l'algèbre $\left\{M_{U}\right\}^{\prime}$ munie de la topologie $w^{*}$ est engendrée par ses idempotents ;

BULLETIN DE LA SOCIÉtÉ MATHÉMATIQUE DE FRANCE 
- $\mu$ est nulle sur les ensembles de Carleson.

Si $S$ désigne le shift unilatéral sur $H^{2}(\mathbb{D})$, nous avons $\{S\}^{\prime}=\mathcal{A}_{S}=H^{\infty}(\mathbb{D})$, d'après le théorème de Sarason. Grâce au théorème de relèvement du commutant de Nagy-Foias, nous en déduisons que $\left\{M_{U}\right\}^{\prime}=\mathcal{A}_{M_{U}}$. Ainsi, tout sous-espace fermé invariant pour $M_{U}$ est en fait hyper-invariant et donc $M_{U}$ est réflexif si et seulement si $M_{U}$ est hyper-réflexif. Par conséquent, $M_{U}$ est hyper-réflexif si et seulement si $\mu$ annule les ensembles de Carleson.

Obtenir l'hyper-réflexivité de $S_{\omega}$ dans le cas dissymétrique revient donc à prouver l'existence d'une fonction intérieure singulière $U$ qui vérifie les deux conditions suivantes :

- il existe $\sigma \in \mathcal{G}(\omega)$ tel que $\int_{0}^{1} K_{U}(r)^{2} / \sigma^{2}(r) \mathrm{d} r<+\infty$;

- la mesure associée à $U$ annule les ensembles de Carleson.

\section{Résolution du problème}

Proposition 3. - Soient $\omega$ un poids dissymétrique et $\sigma \in \mathcal{G}(\omega)$. Il existe alors une fonction intérieure singulière $U$ telle que

$$
\int_{0}^{1} \frac{K_{U}(r)^{2}}{\sigma^{2}(r)} \mathrm{d} r<+\infty \quad \text { et } \sup _{z \in \mathbb{D}} \frac{1-|z|}{|U(z)|}<+\infty .
$$

Démonstration. - Il est bien connu qu'il existe une fonction $u:[0,1[\rightarrow$ ] $0,+\infty[$ telle que

1) $u$ est strictement croissante,

2) $\lim _{r \rightarrow 1^{-}} u(r)=+\infty$,

3) $\int_{0}^{1} u(r) / \sigma^{2}(r) \mathrm{d} r<+\infty$.

Pour $r \in[0,1[$, on pose

$$
f(r)=\min \left(u(r),(1-r)^{-2}\right) .
$$

Il est alors clair que $f$ vérifie les conditions 1 ), 2) et 3) ci-dessus.

D'après le lemme 5.6 de [6], il existe une fonction intérieure singulière $U$ telle que

$$
\sup _{z \in \mathbb{D}} \frac{f^{-1 / 2}(|z|)}{|U(z)|}<+\infty .
$$

Donc il existe $\lambda>0$ tel que

$$
K_{U}(r) \leq \lambda f^{1 / 2}(r) \quad \text { pour } \quad r \in[0,1[.
$$

Ainsi,

$$
\int_{0}^{1} \frac{K_{U}(r)^{2}}{\sigma^{2}(r)} \mathrm{d} r \leq \lambda^{2} \int_{0}^{1} \frac{f(r)}{\sigma^{2}(r)} \mathrm{d} r<+\infty .
$$

TOME $130-2002-\mathrm{N}^{\mathrm{O}} 4$ 
De plus, la relation (2) et la définition de $f$ impliquent

$$
\sup _{z \in \mathbb{D}} \frac{1-|z|}{|U(z)|}<+\infty
$$

Proposition 4. - Soit U une fonction intérieure singulière telle que

$$
\sup _{z \in \mathbb{D}} \frac{1-|z|}{|U(z)|}<+\infty .
$$

Alors la mesure $\mu$ associée à $U$ annule les ensembles de Carleson, et $M_{U}$ est hyper-réflexif.

Démonstration. - Soit $F$ un ensemble de Carleson et $\nu=\mu_{\mid F}$. Supposons que $\nu \neq 0$.

Considérons la fonction intérieure singulière $\theta$ associée à $\nu$. Alors $M_{\theta}$ est une contraction complètement non unitaire et $\sigma\left(M_{\theta}\right) \subset F$. D'après [8], p. 114-117, on a pour $z \in \mathbb{D}$

Or, pour $z \in \mathbb{D}$,

$$
\left\|\left(M_{\theta}-z I\right)^{-1}\right\| \leq \frac{2}{1-|z|} \cdot \frac{1}{|\theta(z)|} .
$$

$$
\left(M_{\theta}-z I\right)^{-1}=\sum_{n \geq 0} z^{n} M_{\theta}^{-n-1}
$$

Grâce aux inégalités de Cauchy, on obtient, pour $n \geq 1$ et $r \in[0,1[$,

$$
\left\|M_{\theta}^{-n}\right\| \leq \frac{2 r^{-n+1}}{1-r} \sup _{|z|=r} \frac{1}{|\theta(z)|},
$$

Comme $|\theta(z)| \geq|U(z)|$ pour $|z|<1$,

$$
\sup _{|z|=r} \frac{1}{|\theta(z)|} \leq \sup _{|z|=r} \frac{1}{|U(z)|}
$$

D'après (4) il existe $K>0$ tel que

Soit $n \geq 1$, alors

$$
\sup _{|z|=r} \frac{1}{|U(z)|} \leq \frac{K}{1-r} \quad \text { pour } \quad r \in[0,1[.
$$

$$
\left\|M_{\theta}^{-n}\right\| \leq 2 K \frac{r^{-n}}{(1-r)^{2}}
$$

pour tout $r \in[0,1[$. Si on prend $r=1-1 / n$, on en déduit que

$$
\left\|M_{\theta}^{-n}\right\|=O\left(n^{2}\right)
$$

car $\lim _{n \rightarrow+\infty}(1-1 / n)^{-n}=$ e. Ainsi $M_{\theta}$ est une contraction dont le spectre est inclus dans un ensemble de Carleson et telle que $\left\|M_{\theta}^{-n}\right\|=O\left(n^{2}\right)$ quand $n$ tend vers l'infini. On en déduit que $M_{\theta}$ est unitaire d'après un théorème 
de J. Esterle [5]. Or ceci est absurde car $M_{\theta}$ est complètement non unitaire. Donc $\nu=0$, et ainsi $\mu$ annule les ensembles de Carleson.

Nous avons donc prouvé le théorème suivant.

THÉORÈme 1. - Si $\omega$ est un poids dissymétrique, le shift $S_{\omega}$ sur $\ell_{\omega}^{2}(\mathbb{Z})$ est hyper-réflexif.

REMARQUE 1. - Si $\omega$ est dissymétrique et décroissant, nous pouvons conclure sans utiliser la proposition 2. Considérons une fonction intérieure singulière $U$ obtenue par la proposition 3. L'opérateur

$$
\widetilde{S}_{\omega}: f+\widehat{E(U)} \longrightarrow S_{\omega} f+\widehat{E(U)}
$$

est alors une contraction complètement non unitaire de classe $C_{0}$, i.e. il existe $u \in H^{\infty}(\mathbb{D}), u \neq 0$, tel que $u\left(\widetilde{S}_{\omega}\right)=0$; plus précisément,

$$
\left\{u \in H^{\infty}(\mathbb{D}) ; u\left(\widetilde{S}_{\omega}\right)=0\right\}=U H^{\infty}(\mathbb{D}) .
$$

Il découle alors d'un théorème de Nagy, Foias et Bercovici [1] que l'hyperréflexivité de $\widetilde{S}_{\omega}$ est équivalente à la réflexivité de $M_{U}$. Comme $M_{U}$ est hyperréflexif, d'après la proposition 5 , nous avons obtenu l'hyper-réflexivité de $\widetilde{S}_{\omega}$, et par suite l'hyper-réflexivité de $S_{\omega}$.

Remerciements. - L'auteur remercie le Professeur J. Esterle pour son soutien et ses nombreux conseils.

\section{BIBLIOGRAPHIE}

[1] Bercovici (H.), Foias (C.) \& Nagy (B. Sz.) - Reflexive and hyperreflexive operators of class $C_{0}$, Acta Sci. Math (Szeged), t. 43 (1981), pp. 513.

[2] Douglas (R.G.), Shapiro (H.S.) \& Shields (A.) - Cyclic vectors and invariant subspaces for the backward shift operator, Ann. Inst. Fourier, t. 20 (1970), pp. 37-76.

[3] Dussau (X.) - Les shifts à poids de croissance polynomiale sont hyperréflexifs, à paraître.

[4] Esterle (J.) - Apostol's bilateral weighted shifts are hypereflexives, Operator Theory : Advances and Applications, à paraître.

[5] _ Uniqueness, strong forms of uniqueness and negative powers of contractions, Funct. Anal. and Operator Theory, Banach Center Pub., t. 30 (1994), pp. 127-145.

[6] _ Singular inner functions and biinvariant subspaces for disymmetric weighted shifts, J. Funct. Anal., t. 144 (1997), no. 1, pp. 60-104.

TOME $130-2002-\mathrm{N}^{\mathrm{O}} 4$ 
[7] Kapustin (V.V.) - Reflexivity of operators : general methods and a criterion for almost isometric contractions, St. Petersbourg Math. J., t. 4 (1993), no. 2, pp. 319-335.

[8] Nagy (B. Sz.) \& FoiAs (C.) - Harmonic analysis of operators on Hilbert spaces, North-Holland Publishing. Co., Amsterdam, 1970.

[9] SHIELDS (A.L.) - Weighted shift operators and analytic function theory, in Topics in Operator Theory, Math. Surveys, vol. 13, Amer. Math. Society, Providence, R.I., 1974, pp. 49-128. 